\title{
Primary retroperitoneal malignant melanoma: A case report
}

\author{
GUO-BING LIU, GUANG-YAO WU, PRASANNA GHIMIRE and ZAI-PENG ZHANG \\ Department of MRI, Zhongnan Hospital of Wuhan University, Wuhan, P.R. China
}

Received March 12, 2011; Accepted July 22, 2011

DOI: $10.3892 / \mathrm{ol} .2011 .397$

\begin{abstract}
Primary malignant melanoma occurring at an extra cutaneous site is rare. A case of primary malignant melanoma located in the retroperitoneum of an 18-year-old female is presented in this study. Histopathological examination of the tissue biopsies at laparotomy with immunohistochemical stains confirmed a diagnosis of malignant melanoma. Further extensive clinical and radiological investigations proved the retroperitoneum to be the primary site.
\end{abstract}

\section{Introduction}

Malignant melanoma is not uncommon, with incidence in the USA increasing by $4 \%$ every year (1) and an annual $3.86 \%$ increase in the thickest tumor ( $>4 \mathrm{~mm}$ ) category (2). The vast majority of primary malignant melanoma occurs in the skin, followed by the choroid layer of the eyes, under the nail, the leptomeninges, oral cavity, nasal mucosa, pharynx, esophagus, bronchus, vaginal or anorectal mucosa (3). Primary malignant melanoma of the retroperitoneum is extremely rare, particularly in young patients. No cases have been reported in the English literature thus far. In this study, we report a case of primary retroperitoneal malignant melanoma in a young female patient and briefly review the literature.

\section{Case report}

An 18-year-old female presented to the Zhongnan Hospital, China, on January 19th, 2006, with a six-month history of a progressively enlarging mass in the right upper abdomen. The patient had no history of any fever, vomiting or icterus. The patient's general physical and chest examination were not significant. Abdominal examination revealed a mildly tender lump in the right hypochondrium of approximately $12 \times 12 \mathrm{~cm}$. The mass was firm in consistency with an indistinct border. The patient's past medical and personal histories were insig-

Correspondence to: Dr Guang-Yao Wu, Department of MRI, Zhongnan Hospital of Wuhan University, 169 East Lake Road, Hubei, Wuhan, P.R. China

E-mail:wuguangy2002@yahoo.com.cn

Key words: malignant melanoma, retroperitoneum, computed tomography, magnetic resonance imaging nificant. All of the laboratory investigations, including liver and renal function tests, were within normal limits.

A computed tomography (CT) scan of the abdomen showed an irregular heterogeneous soft-tissue density mass located in the anterior pararenal space inferior to the right lobe of the liver, with the right kidney slightly compressed and bowel loops displaced medially. CT results showed that the mass measured approximately 70x108 mm in the largest section with an indistinct border and was not separable from the gastric sinus and gallbladder (Fig. 1A). Post-contrast CT showed a heterogeneously enhancing mass with marked peripheral enhancement, and a number of tortuous and dilated vessels (Fig. 1B).

The patient underwent total resection of the retroperitoneal mass. Per-operatively, the mass, measuring $10 \times 7 \times 5 \mathrm{~cm}$, was seen extending superiorly to the inferior border of the liver, inferiorly to the level of the iliac crest, and crossing the midline medially, with certain areas enclosing the abdominal aorta and compressing the inferior vena cava. The mass was encapsulated, with the capsule absent at certain areas with tortuous and dilated vessels on its surface. The tumor was soft and homogeneously black and fleshy on the cut surface, with a tendency to bleed.

Histopathological analysis of the tissue biopsies disclosed a dense proliferation of epithelioid, polygonal neoplastic cells with abundant eosinophilic cytoplasm, round to ovoid nuclei and prominent nucleoli, arranged in a uniform nested to fascicular pattern of growth, separated by vascularized fibrocollagenous septa (Fig. 2A and B). Focal tumor cells stained strongly positive by immunohistochemistry for S-100, HMB-45 and melanin A, and mildly positive for tyrosinase (Fig. 3A-C). A Fontana-Masson stain confirmed the presence of melanin pigment.

A thorough laboratory and radiological investigation was performed to locate any primary or secondary deposits in the liver and brain; no other abnormalities were detected. A diagnosis of primary retroperitoneal melanoma was then made following careful dermatological and ophthalmological examination, which ruled out the presence of cutaneous or choroidal melanoma.

The immediate postoperative course of the patient was uneventful and she was discharged. A follow-up CT examination performed nine months later showed three metastatic nodular lesions in the liver (Fig. 4A and B). Although regular chemotherapy (6 cycles of DDBT regimen: dacarbazine, DTIC; cisplatin, DDP; carmustine, BCUN; and tamoxifen, TAM) was administered, the metastasis progressed, as shown on the 
A

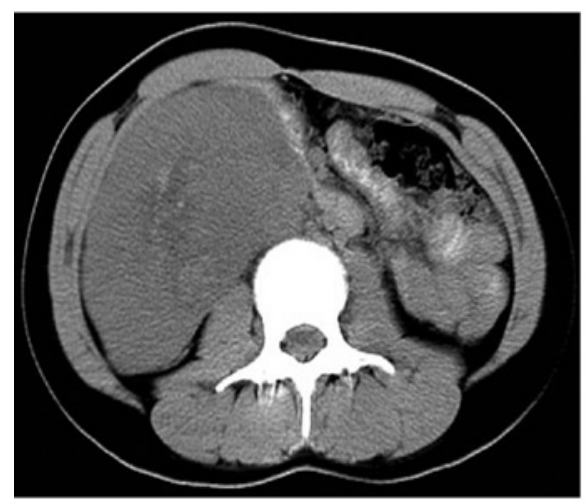

B

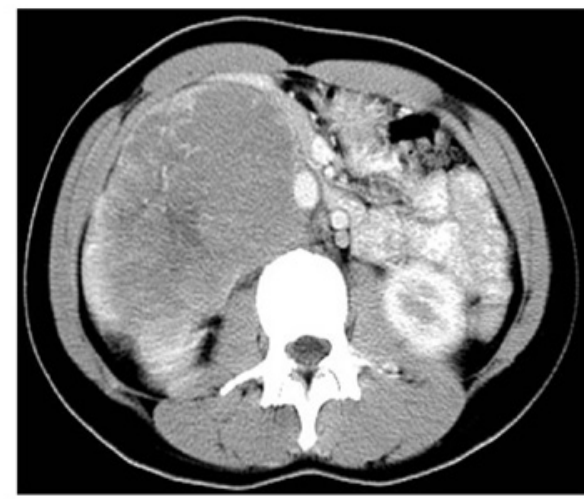

Figure 1. (A) Precontrast-enhanced and (B) postcontrast-enhanced CT images show a retroperitoneal mass with heterogeneous enhancement and tortuous blood vessels surrounding it.

A

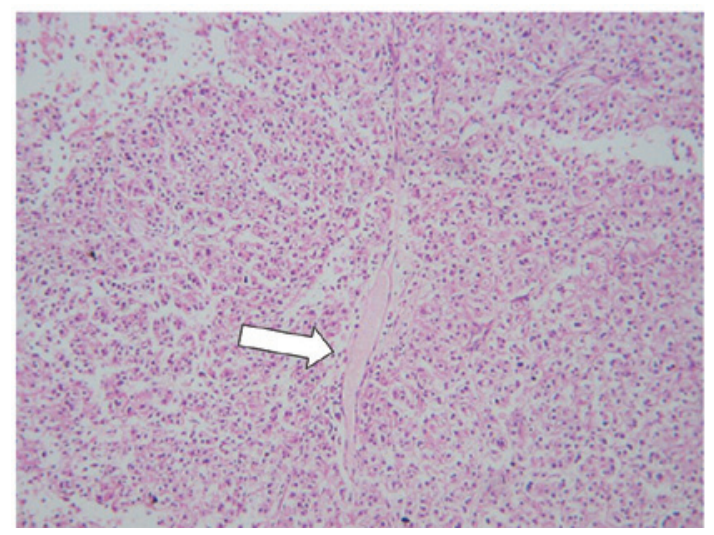

B

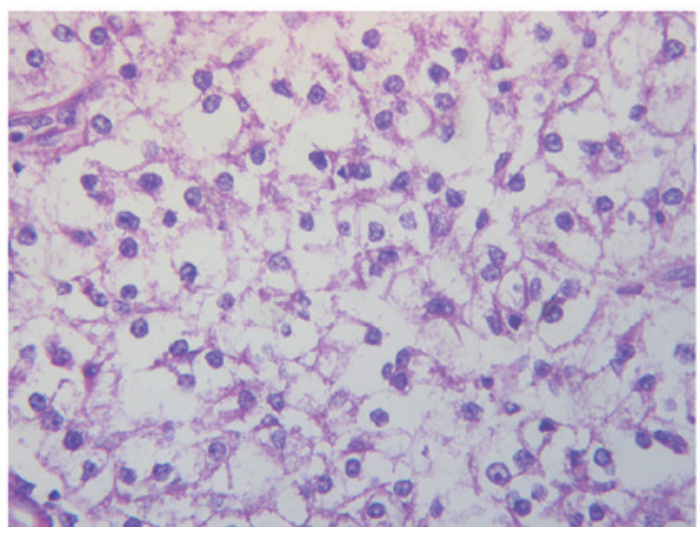

Figure 2. (A) H\&E, magnification, x100. (B) H\&E , magnification, $x 400$. A photomicrograph of the operated specimen shows epithelioid neoplastic cells with abundant eosinophilic cytoplasm, round to ovoid nuclei and prominent nucleoli, arranged in a uniform nested to fascicular pattern of growth, separated by vascularized fibrocollagenous septa (white arrow).

A

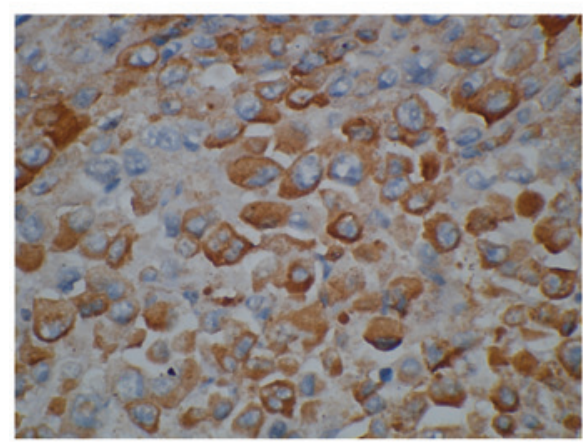

B

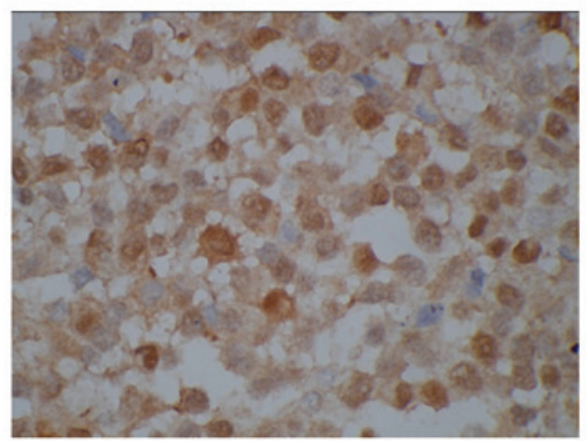

C

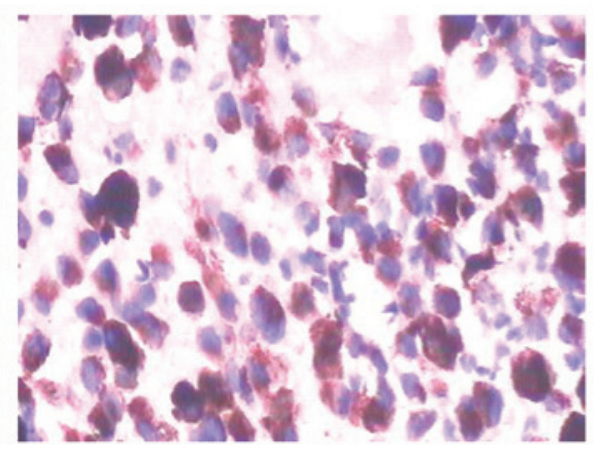

Figure 3. Immunohistochemistry of the specimen shows cells staining positive for (A) HMB-45 (magnification, x400), (B) S-100 (magnification, x400) and (C) melanin A (magnification, x400).

abdominal CT taken on February 20th, 2008 (Fig. 5A-D). The patient succumbed to the disease in December 2008, with a survival interval from the initial diagnosis of approximately 23 months.

\section{Discussion}

Accounting for $3 \%$ of all primary cutaneous malignancies (4), malignant melanoma primarily presents as a cutaneous lesion, with the overall mean age of presentation in the 6th decade, with a slight predilection in men (5). However, less than $10 \%$ of all cases arise from non-cutaneous regions (3), which may be caused by unknown cytokines excreted from cells of the skin when exposed to ultraviolet rays, or by unknown injurious factors (6).

Malignant melanoma primarily occurring in the retroperitoneum at a young age has not been previously reported in the English literature. Our patient was extensively investigated 
A

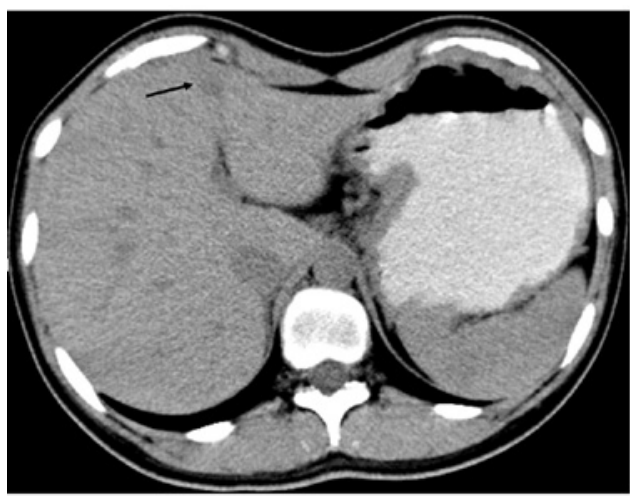

B

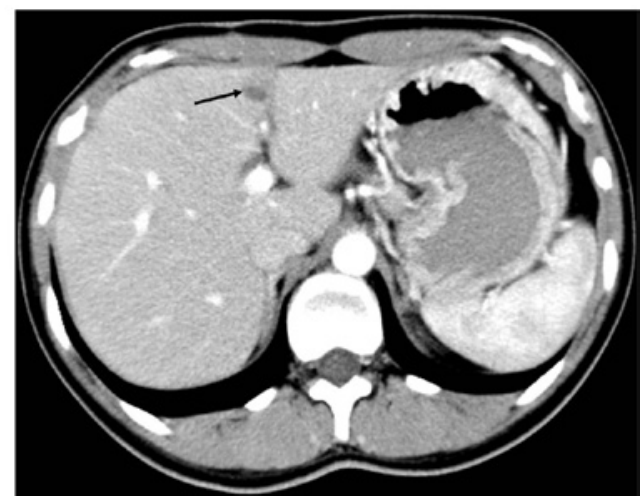

Figure 4. (A) Precontrast-enhanced and (B) postcontrast-enhanced CT images, taken 9 months after the patient was discharged, demonstrated a metastatic focus (black arrow).

A

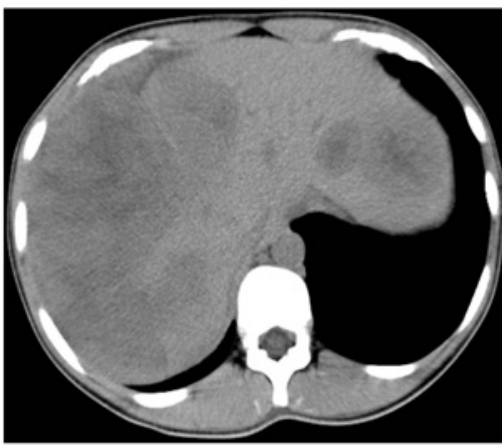

C

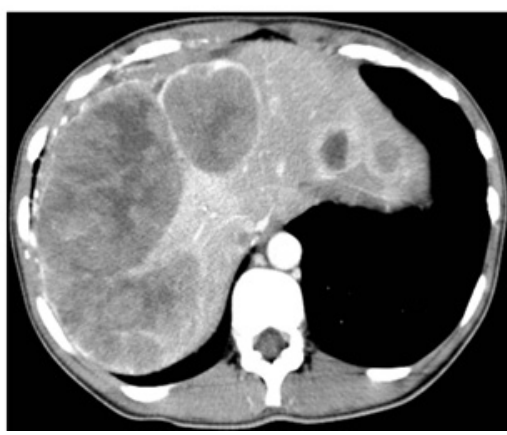

B

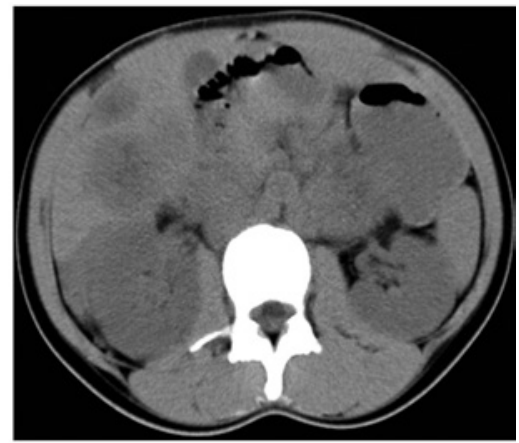

D

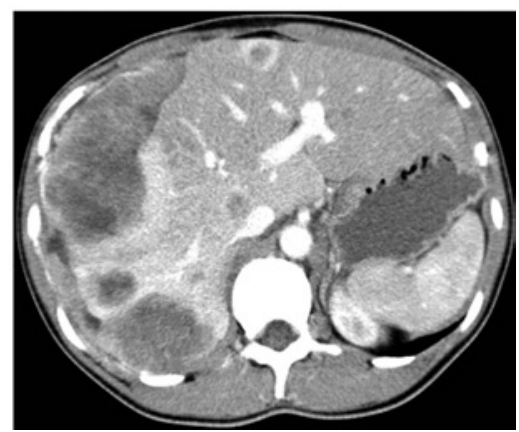

Figure 5. (A and B) Precontrast-enhanced CT images of different sections and (C and D) corresponding postcontrast-enhanced CT images, taken in February 2008 , show the metastasis to have progressed, compared to previous scans.

at the time of diagnosis. However, no other sites showed evidence of tumor or nevus, thus confirming the retroperitoneum as the primary site of involvement. Melanoma is thought to arise where melanocytes reside, including the sympathetic chain, which originates from the neural ectoderm, as well as the autonomic ganglion cells (7). A few cases of malignant melanoma have been reported to occur in the posterior mediastinum, derived from the melanocytes scattered in the sympathetic chain and autonomic nerve plexuses (7-9). We postulate that the tumor of the current patient also originated from the sympathetic chain or autonomic nerve plexuses of the retroperitoneum, which potentially connected to its counterpart in the mediastinum.

Radiological examination is useful in the diagnosis of malignant melanoma. CT is useful as a common tool to detect focal retroperitoneal lesions, to rule out metastasis, to guide percutaneous biopsies and to demonstrate response to therapy.
CT is employed to evaluate the margin, size and density of a tumor mass, and particularly the pattern of enhancement, as malignant melanoma is usually immersed in blood and surrounded by blood vessels, which cause prominent enhancement and tortuous vessels on post-contrast CT, as was evident in this patient. The MRI findings are variable, but relatively specific, depending on the type of melanoma, the content of melanin within the tumor regions and the presence or absence of intratumoral hemorrhage (10-12). Isiklar et al (11) divided melanoma into four patterns: i) melanotic pattern, the typical type of melanoma, comprising high signal intensity on T1-weighted images and low signal intensity on T2-weighted images; ii) amelanotic pattern, another commonly described type, which is hypointense or isointense on T1-weighted images and hyperintense or isointense on T2-weighted images; iii) mixed pattern, which presents between a melanotic and amelanotic pattern; and iv) hemorrhagic pattern, which has a 
signal intensity that is related to an associated hematoma. An MRI scan, which would have added diagnostic value in this particular case, was not performed.

Histopathological and immunohistochemical analyses are indispensable to the diagnosis of melanoma. Pathologically, tumor cells may be classified into four types: i) epithelioid-cell pattern, consisting of enlarged round cells, with large round to oval, hyperchromatic to vesicular nuclei, eosinophilic nuclear inclusions, prominent eosinophilic nucleoli and a variable amount of eosinophilic to clear-appearing cytoplasm, which are always arranged in compact nests or cords, as shown in this patient; ii) spindle-cell pattern, consisting of a fascicular to storiform growth pattern composed of elongated to oval cells characterized by cellular pleomorphism, multinucleated cells and increased mitotic activity. The nuclei are large, vesicular to hyperchromatic with prominent nucleoli, and have a variable amount of eosinophlic cytoplasm; iii) lymphoma-like pattern, composed of cells with round, eccentrically located, vesicular to basophilic appearing nuclei and eosinophilic cytoplasm; and iv) pleomorphic pattern $(13,14)$.

Extra-cutaneous melanomas exhibit the same immunohistochemical and ultrastructural features as their cutaneous counterparts. S-100 protein, HMB-45, melanin A (also knownas MART-1), vimentin and occasionally anti-tyrosinase antibody are used as immunohistochemical tools in the diagnosis of melanoma (14-18). S-100 has the highest sensitivity for the differentiation of melanocytes. However, as a protein affecting the trafficking of intracellular calcium, S-100 is also found in a number of other tissues, including nerve sheath tumors and gliomas, neuroendocrine cells, certain histiocytic proliferations, Langerhans cells and, rarely, in poorly differentiated carcinomas. S-100 is thus preferentially used as a screening tool $(14,17)$. HMB-45 and melanin A are the two most common 'melanocyte-specific' monoclonal antibodies used in the diagnosis of melanoma, as they are specific to the inner membrane proteins present on premelanosomes, noted exclusively in cells that show any type of melanocytic differentiation. The sensitivity of HMB-45 for melanoma is reported to reach up to $93-100 \%$, with a similar distribution as melanin A $(15,16,18)$. Vimentin is uniformly present in malignant melanoma with a reported sensitivity of 93\% (14). Although less widely used, anti-tyrosinase antibodies are highly specific and sensitive for melanocytic neoplasms, having sufficient maturation with a sensitivity of approximately $86 \%$ (14). The presence of melanin pigment is favorable to the diagnosis of malignant melanoma, but it is not essential, as approximately $50 \%$ of cases are negative for the melanin pigment.

When considering the differentiation of malignant melanoma, metastasis from a cutaneous melanoma is the most likely of the differential diagnoses. Approximately $90 \%$ of melanoma patients present with secondary lesions, of which $45 \%$ have radiological evidence, and in $10 \%$ of cases, the primary site is unknown (19). Thus, extensive investigation is required when facing a single lesion of melanoma. Other differential diagnoses include pigmented extra-adrenal paraganglioma $(20,21)$, melanotic Schwannoma (22) and other neuroectodermal neoplasms (23-25), which may be excluded by negative immunohistochemical stains for neuroendocrine markers, e.g., NSE, chromogranin and synaptophysin (26).
Melanoma is a lethal disease, with a clear character and an unpredictable clinical course. Complete resection of the lesion is the principal treatment for melanoma, which although only palliative, does not prevent further metastasis. The role of adjuvant chemotherapy has yet to be established, with no clear evidence of improved survival. However, in patients with multiple metastasis, it is always the main management modality. In recent years, immunotherapy and molecular-targeted therapy have played a more pivotal role in the treatment of malignant melanoma (27-30). The prognosis of malignant melanoma is dismal, particularly extra-cutaneous lesions, which are more aggressive than their cutaneous counterparts. The median survival for primary mucosal melanoma was reported to be from 23 to 48 months after the initial lesions were resected (31-33). The survival interval of our patient was 23 months, suggesting a relatively worse prognosis in a primary melanoma involving the retroperitoneum.

In conclusion, we have followed up and reported a pathologically confirmed case of primary malignant melanoma located in the retroperitoneum, which is extremely rare due to its atypical location and the relatively young age of presentation.

\section{References}

1. Rager EL, Bridgeford EP and Ollila DW: Cutaneous melanoma: Update on prevention, screening, diagnosis, and treatment. Am Fam Physician 72: 269-276, 2005.

2. Linos E, Swetter SM, Cockburn MG, Colditz GA and Clarke CA: Increasing burden of melanoma in the United States. J Invest Dermatol 129: 1666-1674, 2009.

3. Chang AE, Karnell LH and Menck HR: The National Cancer Data Base report on cutaneous and noncutaneous melanoma: a summary of 84,836 cases from the past decade. The American College of Surgeons Commission on Cancer and the American Cancer Society. Cancer 83: 1664-1678, 1998.

4. Capizzi P and Donohue J: Metastatic melanoma of the gastrointestinal tract: a review of the literature. Compr Ther 20: 20-23, 1994.

5. Poos HPAM, Kruijff S, Bastiaannet E, Van Ginker RJ and Hoekstra HJ: Therapeutic groin dissection for melanoma: Risk factors for short term morbidity. Eur J Surg Oncol 35: 877-883, 2009.

6. Yamaguchi T, Shionki Y, Koide K and Kurioka H: A case of primary malignant melanoma of the esophagus and analysis of 193 patients in Japan. Nippon Shokakibyo Gnkkal Zasshi 101: 1087-1094, 2004.

7. Karuppiah SV and Buchan KG: Primary malignant melanoma: a rare cause of mediastinal mass. Jpn J Thorac Cardiovasc Surg 54: 396-398, 2006.

8. Lau CL, Bentley RC, Gockerman JP, Que LG and D'Amico TA: Malignant melanoma presenting as a mediastinal mass. Ann Thorac Surg 67: 851-852, 1999.

9. Shishido M, Nagao N and Miyamoto K: Mediastinal amelanotic melanoma presenting as superior vena cava syndrome. Nihon Kyobu Shikkan Gakkai Zasshi (Japanese Journal of Thoracic) 35: 240-244, 1997.

10. Lemke AJ, Hosten N, Bornfeld N, et al: Uveal melanoma: correlation of histopathologic and radiologic findings by using thin-section MR imaging with a surface coil. Radiology 210: 775-783, 1999.

11. Isiklar I, Leeds NE, Fuller GN and Kumar AJ: Intracranial metastatic melanoma: correlation between MR imaging characteristics and melanin content. AJR Am J Roentgenol 165: 1503-1512, 1995.

12. Escott EJ: A variety of appearances of malignant melanoma in the head: A review. Radiographics 21: 625-639, 2001.

13. Wenig BM: Laryngeal mucosal malignant melanoma: a clinicopathologic, immunohistochemical, and ultrastructural study of four patients and a review of the literature. Cancer 75: 1568-1577, 1995 
14. Chute DJ, Cousar JB and Mills SE: Anorectal malignant melanoma morphologic and immunohistochemical features. Am J Clin Pathol 126: 93-100, 2006.

15. Jungbluth AA, Busam KJ, Cerald WL, et al: A103: An anti-melan-a monoclonal antibody for the detection of malignant melanoma in paraffin-embedded tissues. Am J Surg Pat hol 22: 595-602, 1998.

16. Busm KJ, Chen YT, Old LJ, et al: Expression of melan-A (MART1) in benign melanocytic nevi and primary cutaneous malignant melancma. Am J Surg Pathol 22: 976-982, 1998.

17. Busam KJ, Iversen K, Coplan KC and Jungbluth AA: Analysis of microphthalmiat ranscription factor expression in norma tissues and tumors, and comparison of it $\mathrm{s}$ expression with S-100 protein, gp100, and tyrosinase in desmorlastic malignant melanoma. Am J Surg Pathol 25: 197-204, 2001.

18. De-Vries TJ, Smeets M, de-Graaf R and Hou-Jensen K Expression of gp100, MART-1, tyrosinase, and S100 in paraffin-embedd primary melanomas and locoregional, lymph node, and visceral metastases: implications for diagnosis and immunotherapy. A study conducted by the EORTC Melanoma Cooperative Group. J Pathol 193: 13-20, 2001.

19. Greco CS, Soffietti R, Bradac GB and Boldorini R: Primitive cerebral melanoma: case report and review of the literature. Surg Neurol 55: 163-168, 2001.

20. Lack EE, Kim H and Reed K: Pigmented ('black') extraadrenal paraganglioma. Am J Surg Pathol 22: 265-269, 1998.

21. Moran CA, Albores-Saaverda J, Wenig BM and Mena $\mathrm{H}$ Pigmented extraadrenal papraganglions. A clinicopathologic and immunohistochemical study of five cases. Cancer 79: 398-402, 1997.

22. Marco V, Sirvent J, Alvarez Moro J, Clavel M, Muntal MT and Bauza A: Malignant melanotic schwannoma fine-needle aspiration biopsy findings. Diagn Cytopathol 18: 284-286, 1998.
23. Matsumine A, Kusuzaki K, Nakamura T, et al: Differentiation between neurofibromas and malignant peripheral nerve sheath tumors in neuro-fibromatosis 1 evaluated by MRI. J Cancer Res Clin Oncol 135: 891-900, 2009

24. Li CS, Huang GS, Wu HD, et al: Differentiation of soft tissue benign and malignant peripheral nerve sheath tumors with magnetic resonance imaging. Clin Imaging 32: 121-127, 2008.

25. Stoll G, Bendszus M, Perez J and Pham M: Magnetic resonance imaging of the peripheral nervous system. J Neurol 256: 1043-1051, 2009.

26. Vlodavsky E, Ben-Izhak O, Best LA and Kerner H: Primary Malignant Melanoma of the Anterior Mediastinum in a Child. Am J Surg Pathol 24: 747-749, 2000.

27. Garbe C, Eigentler T, Keilholz U, Hauschild A and Kirkwood J: Systematic Review of Medical Treatment in Melanoma: Current Status and Future Prospects. Oncologist 16: 5-24, 2011.

28. Ko JM and Fisher DE: A new era: melanoma genetics and therapeutics. J Pathol 223: 241-250, 2011.

29. Kim CJ, Dessureault S, Gabrilovich D, Reintgen DS and Slingluff CL: Immunotherapy for melanoma. Cancer Control 9 : 22-30, 2010

30. Sivendran S, Glodny B, Pan M, Merad M and Saenger Y: Melanoma immunotherapy. Mt Sinai J Med 77: 620-642, 2010.

31. Schuchter LM, Green R and Fraker D: Primary and metastatic diseases in malignant melanoma of the gastrointestinal tract. Curr Opin Oncol 12: 181-185, 2000.

32. Kim W, Baek JM, Suh YJ, Jeon HM and Kim JA: Ileal malignant melanoma presenting as a mass with aneurysmal dilatation: a case report. J Korean Med Sci 19: 297-301, 2004.

33. Berger AC, Buell JF, Venzon D, Baker AR and Libutti SK: Management of symptomatic malignant melanoma of the gastrointestinal tract. Ann Surg Oncol 6: 155-160, 1998. 